\title{
From elastic deformation to flow in tempered chocolate
}

DOI:

$10.1122 / 1.5038253$

\section{Document Version}

Accepted author manuscript

Link to publication record in Manchester Research Explorer

\section{Citation for published version (APA):}

Bergemann, N., Heil, M., Smith, B., \& Juel, A. (2018). From elastic deformation to flow in tempered chocolate. Journal of Rheology, 62(1187). https://doi.org/10.1122/1.5038253

\section{Published in:}

Journal of Rheology

\section{Citing this paper}

Please note that where the full-text provided on Manchester Research Explorer is the Author Accepted Manuscript or Proof version this may differ from the final Published version. If citing, it is advised that you check and use the publisher's definitive version.

\section{General rights}

Copyright and moral rights for the publications made accessible in the Research Explorer are retained by the authors and/or other copyright owners and it is a condition of accessing publications that users recognise and abide by the legal requirements associated with these rights.

\section{Takedown policy}

If you believe that this document breaches copyright please refer to the University of Manchester's Takedown Procedures [http://man.ac.uk/04Y6Bo] or contact uml.scholarlycommunications@manchester.ac.uk providing relevant details, so we can investigate your claim.

\section{OPEN ACCESS}




\title{
From elastic deformation to flow in tempered chocolate
}

\author{
Nico Bergemann ${ }^{1,2}$, Matthias Heil ${ }^{1}$, Beccy Smith ${ }^{3} \&$ Anne Juel $^{2 *}$ \\ ${ }^{1}$ School of Mathematics and Manchester Centre for Nonlinear Dynamics, \\ The University of Manchester, Oxford Road, Manchester, M13 9PL \\ ${ }^{2}$ Manchester Centre for Nonlinear Dynamics and School of Physics and Astronomy, \\ The University of Manchester, Oxford Road, Manchester, M13 9PL \\ ${ }^{3}$ Mondelez International, Bournville Place, Birmingham, B30 2LU
}

(Dated: July 10, 2018)

\begin{abstract}
We characterise the yield behaviour and flow curve of a commercially available, molten, tempered milk chocolate with a fat content of $30 \%$, from rheological measurements performed with a rotational rheometer fitted with a four-bladed vane spindle in a large-gap setup. We show that the yield behaviour can be captured by the viscoelastic White-Metzner model, which indicates that elastic deformation precedes flow as the shear stress is increased. Following an initial fluidisation cycle, the shear modulus of the tempered chocolate increases by a factor sixty to $G=4.4 \times 10^{4} \mathrm{~Pa}$. We find that the Bingham model provides the best fit to our extensive flow curve data, where the yield stress is $\tau_{\text {yield }}=36.1 \pm 0.6 \mathrm{~Pa}$ and the Newtonian plateau viscosity is $\mu_{B}=8.7 \pm 0.02 \mathrm{~Pa}$ s.
\end{abstract}

\footnotetext{
*anne.juel@manchester.ac.uk
} 


\section{INTRODUCTION}

The measurement of the rheological properties of yield stress fluids is a complex issue which has been the subject of extensive research in recent years [1,2]. The yield stress marks the threshold between solid viscoelastic behaviour and liquid behaviour characterised by a non-Newtonian constitutive relation, such as the Herschel-Bulkley model for shear-thinning materials [3]. However, this solid-liquid transition is strongly dependent on the microscopic structure of the material, and may feature unsteady flows and complexity such as shear bands and wall slip, which can lead to erroneous interpretation of macroscopic data [2].

These complicating features primarily affect start-up flows [4] and are typically not observed when decreasing the shear stress in quasi-steady flow [5]. The determination of the true value of the static yield stress may require long waiting times, although in practice, a more relevant apparent static yield stress will be determined by the time-scale of the experiment [2]. The aim of this paper is to characterise the constitutive behaviour of molten tempered chocolate, a yield-stress fluid, on moderate time scales, so that, for example, it may be incorporated into a viscoelastic or generalised Newtonian numerical model to predict the fluidisation of tempered chocolate by mechanical vibration, a technique commonly employed on industrial production lines.

Molten chocolate consists of sub-millimetric solid cocoa $(<50 \mu \mathrm{m})$ and sugar particles suspended in a continuous phase of cocoa butter; with additional solid milk powder particles suspended in milk chocolate [6]. The total solid content of chocolate is $65-75 \%$ [7], making it a dense colloidal suspension. The particles increase the apparent viscosity of the suspending liquid and their flow-dependent distribution results in the non-Newtonian behaviour of the suspension. Interparticle forces can lead to the formation of a continuous network which spans the entire suspension [1]. This interaction network breaks down when shear stress is applied, resulting in flow, but it builds up at rest, and is responsible for the presence of a yield stress [8]. Most rheological measurements have been conducted on untempered chocolate where the temperature is sufficiently high for the cocoa butter not to crystallise $[7,9-13]$.

The tempering of chocolate is crucial for the quality of the final product in terms of thermal contraction (necessary for release from the mould), snap, gloss and mouth feel [1416]. The continuous phase, cocoa butter, is formed of long-chain organic acids, referred 
to as fatty acids. The main components of cocoa butter are triglycerides which consist of three fatty acids attached to a glycerol backbone. Cocoa butter crystallises upon cooling to temperatures below the melting point, resulting in the formation of six different crystal structures, with distinct melting points and densities [17], a characteristic known as polymorphism. Polymorphic crystal structures with low melting temperatures are not stable, so that chocolate composed of unstable polymorphs (types I to IV) or a mixture of polymorphs (types I to VI) spontaneously undergoes physical changes, which degrade the structure and appearance of the chocolate. A common example is fat bloom, a white coating on the surface of solid chocolate. The tempering process enables the formation of a sufficient number of stable type $\mathrm{V}$ crystals and disperses them uniformly within the chocolate through combined heating/cooling and shearing. It consists of a cooling phase during which the less stable polymorphs I to IV are formed, followed by controlled heating of the melt to promote the transformation of these polymorphs into the stable form $\mathrm{V}$ [14]. In industrial chocolate manufacturing the molten chocolate is tempered before it is deposited in moulds to form the required shape. Therefore, understanding the flow behaviour of tempered chocolate is important to model and optimise the production process.

Briggs and Wang [15] showed that rheological data may be used to infer the influence of shear rate and shearing time on the crystallization of chocolate indirectly through changes in apparent viscosity. They found that the Herschel-Bulkley model provides a better fit to the flow curve than the Casson model which is widely applied by the chocolate industry. Long-standing concerns over the suitability of the Casson model to describe untempered chocolate $[9,18]$ have led to modifications of the model and new methods of rheological analysis $[10,12]$. In this paper, we present carefully controlled measurements of the steady flow curve of tempered chocolate recorded in a vane rheometer (see Figure 1) and show that it is best fitted by the Bingham model, a special case of the Herschel-Bulkley model where the shear-thinning power index is one. Above the yield stress, the viscosity steeply decreases with increasing shear rate and approaches a Newtonian plateau for values of the shear rate $\dot{\gamma} \gtrsim 40 \mathrm{~s}^{-1}$; this behaviour was also found by Briggs and Wang [15] for a different type of tempered chocolate for $\dot{\gamma} \gtrsim 70 \mathrm{~s}^{-1}$.

The solid behaviour of untempered molten chocolate has been shown by Taylor et al. [12] to result from interactions of the densely packed particles in suspension, associated with an shear modulus measured by oscillatory shear rheometry in excess of $10^{4} \mathrm{~Pa}$. Here, we focus 
on the yield behaviour of tempered chocolate for successive cycles of prescribed increase and decrease of the applied stress using a vane rheometer (see Figure 1), and find that a simple viscoelastic model, the White-Metzner model [19], adequately captures the variation of strain rate with stress. Dinkgreve et al. [20] recently showed that simple viscoelastic models can also capture features of the time dependence of viscosity in the vicinity of the yield point. We observe an increase in the apparent static yield stress across cycles associated with the stiffening of the material, so that the apparent static yield stress exceeds the dynamic yield stress, consistent with recent developments in the understanding of yielding in dense suspensions $[4,5,21]$.

The paper is organised as follows. The experiments and data processing methods are described in §II. Results are presented in §III where the transition from viscoelastic solid to flow behaviour is modelled using the White-Metzner viscoelastic model in §III A. The rheological parameters for our tempered chocolate are then determined by fitting the Bingham model to the flow curve in §IIIB.

\section{EXPERIMENTAL METHODS}

\section{A. Experimental apparatus}

We performed rheological measurements on a commercially available, tempered milk chocolate with a fat content of $30 \%$ using a Brookfield R/S-Plus (SST) rheometer. The temporal resolution of the rheometer is one measurement per second and it can be operated by controlling torque and measuring angular velocity, or vice-versa. The parameter range we investigated was $0<\omega \leq 10 \pi \mathrm{rad} / \mathrm{s}$ and $0<M \leq 10 \mathrm{mN}$.m for angular velocity and torque, respectively. As shown schematically in Figure 1(a), we used a four-bladed vane spindle rotating in a stationary cylindrical glass beaker of inner radius $R_{\text {cup }}=42.5 \pm 0.25 \mathrm{~mm}$, filled with tempered chocolate. We placed the beaker in a circulating water bath (Brookfield TC-150) at a temperature of $29.0 \pm 0.1^{\circ} \mathrm{C}$, which was chosen to be marginally higher than the temperature of the chocolate of $28.2 \pm 0.3{ }^{\circ} \mathrm{C}$ at the outlet of the temperer (see $\S I I B$ ) [11]. In addition, we fitted an insulating lid on the beaker, with a hole for the spindle, in

order to minimise heat loss at the free surface, thus helping to maintain the chocolate in its tempered state. These precautions were essential to achieve reproducible measurements. 


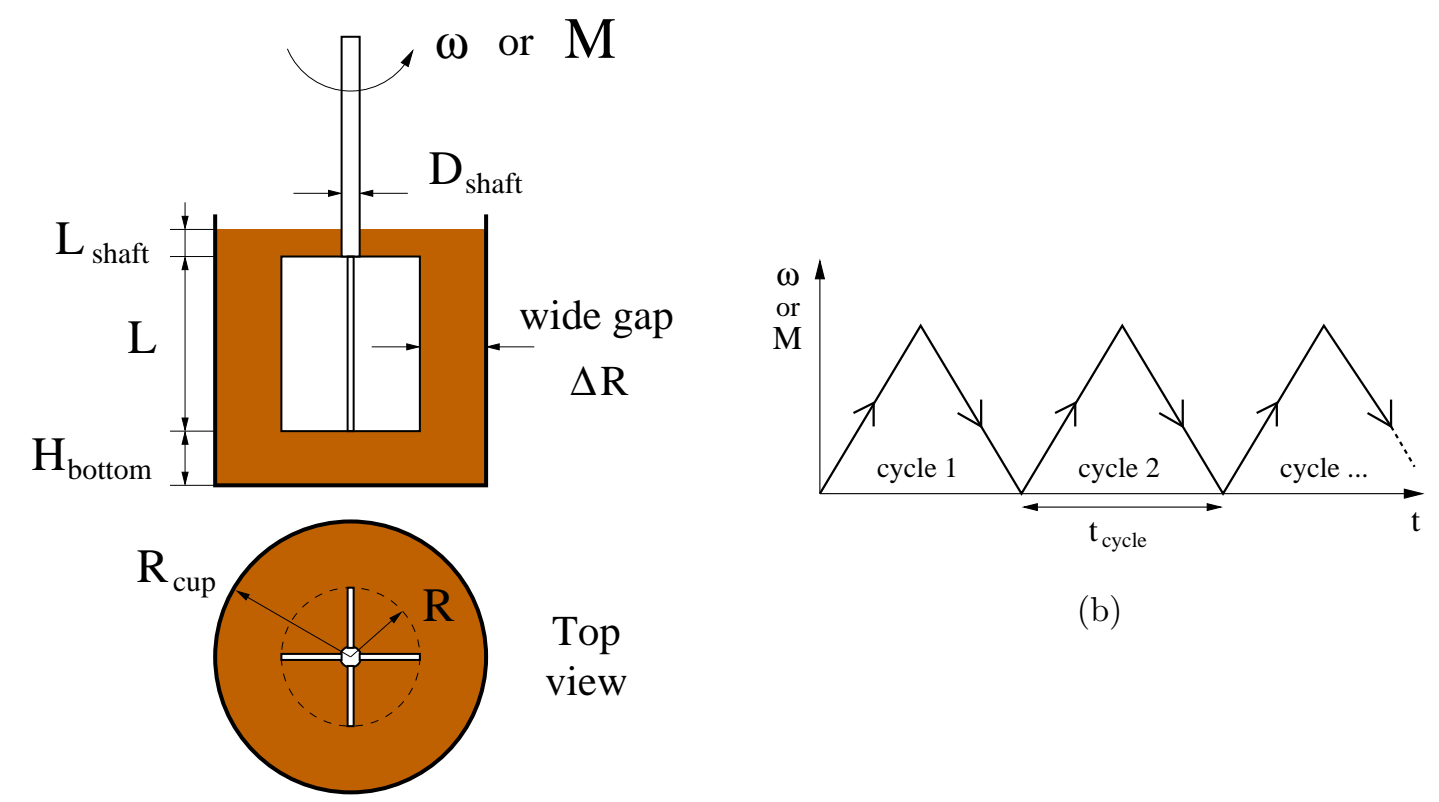

(a)

FIG. 1. Schematic diagram (not to scale) of (a) the coaxial vane geometry, where the four-blade vane spindle rotates and the outer cylinder (cup) is stationary, and (b) the cyclic form of the imposed angular velocity or torque.

We used two vane spindles with blades of different length and obtained consistent results. Each spindle of radius $R=10 \pm 0.01 \mathrm{~mm}$ consisted of four rectangular blades of thickness $1 \pm 0.01 \mathrm{~mm}$ and length either $L=20 \pm 0.01 \mathrm{~mm}$ (short) or $40 \pm 0.01 \mathrm{~mm}$ (long). These were rigidly mounted around a central shaft of diameter $D_{\text {shaft }}=4 \pm 0.01 \mathrm{~mm}$, as shown in Figure 1(a). The use of a vane spindle, instead of the classical concentric cylinder geometry, minimises slippage because of the presence of stationary fluid trapped between the blades $[22,23]$. Slippage at the beaker wall was minimised by using a large gap between the spindle and the outer wall and by roughening the inner boundaries of the glass beaker, which we coated with sub-millimetre glass beads on a layer of epoxy resin.

\section{B. Experimental protocol}

We tempered the chocolate using a Model Prima tempering machine (FBM, Italy). Prior to each rheological measurement we determined the temper level of the chocolate using an EXOTHERM 7400 temper meter (Systech Analytics SA, Switzerland), which assesses the level of temper by recording a cooling curve that correlates with the development of seed 
crystals [16]. In all our experiments, the crystallisation temperature at the point of inflexion of the cooling curve was found to be between 18.0 and $22.0^{\circ} \mathrm{C}$, and the Temper Index was between 4 and 6 , indicating a well-tempered state. We then filled the glass beaker with liquid chocolate from the outlet of the tempering machine to heights of $50 \pm 2 \mathrm{~mm}$ and $70 \pm 2 \mathrm{~mm}$ for use with the short and long spindle vanes, respectively. We gently tapped the fluid-filled beaker on a smooth surface to remove any air bubbles and to level the free surface. This operation was only performed for approximately ten seconds in order to avoid excessive cooling at the surface. The filling heights were chosen so that the distance from the bottom of the spindle to the bottom wall of the container remained constant at a relatively large value $H_{\text {bottom }}=20 \pm 3 \mathrm{~mm}$ to reduce the influence of the bottom boundary. We immersed the vane spindle to a depth of $L_{\text {shaft }}=10 \pm 1 \mathrm{~mm}$ in the centre of the beaker with a radial variation of $\pm 2 \mathrm{~mm}$, so that the radial gap was $\Delta R=32.5 \pm 2 \mathrm{~mm}$. This immersion depth was sufficient to prevent the spindle from being exposed and to minimise deformation of the free surface during experimentation. We then pre-sheared the chocolate at a low constant angular velocity of $\pi / 2 \mathrm{rad} / \mathrm{s}$ for ten seconds to level the free surface deformed by the immersion of the spindle, without interfering with the microscopic structure of the chocolate. After a rest period of one second, we applied shear cyclically by imposing either the angular velocity or the torque as shown schematically in Figure 1(b). The rate of increase (decrease) was set by the cycle period, $t_{\text {cycle. }}$ We performed two types of measurements in order to assess the flow and yield behaviour of tempered chocolate. When investigating flow behaviour, we used the short spindle, because it allowed the measurement of the largest range of shear stress values within the maximum torque rating of the rheometer, $M_{\max }=10 \mathrm{mN} . \mathrm{m}$. We applied cycle periods of 30,60, 120, and $240 \mathrm{~s}$, in order to assess the influence of the rate of increase/decrease of either rotation rate or torque on the rheological behaviour. The reproducibility of our measurements was assessed by repeating the cycles for a total time interval of $600 \mathrm{~s}$, except for the longest cycle period of $240 \mathrm{~s}$, where the total time was $720 \mathrm{~s}$. This maximum experimentation time was selected based on measurements of the temper index in chocolate held at rest at $29^{\circ} \mathrm{C}$, which rose from 4 to 6 over this period of time. The temper index may additionally rise through shear-induced crystallisation [24], but in each of our experiments, the differences between torque and angular velocity values measured in the first and last cycles remained consistently less than 10\%, which was the typical variability between experiments. In order to characterise the yield behaviour, we imposed the torque 
and used the long spindle to maximise the resolution of the imposed shear stress. A long cycle period of 240 s reduced the shear stress increments to $\approx 3 \mathrm{~Pa}$ (about half the size of the smallest increments in the measurements of the flow behaviour), but limited the number of cycles that could be recorded within one experiment to three.

\section{Data processing}

Each experimental data set consisted of discrete measurements of torque [or angular

velocity] as a function of the imposed angular velocity [or torque]. To convert this data into shear stress and shear rate we adopted a procedure suggested by Yeow et al. [23]. The shear stress $\tau$ was readily calculated from the measured or imposed torque and the geometry of the spindle as

$$
\tau(R)=\left.\tau_{r \phi}\right|_{r=R}=\frac{M}{2 \pi R^{2} L_{\mathrm{tot}}}, \quad L_{\mathrm{tot}}=L+L_{0}
$$

where we corrected for spindle end effects by adding a virtual length, $L_{0}=2 R / 3$, to the actual spindle length, as proposed by van Wazer et al. [25]. We assumed that the chocolate between the blades remained un-yielded, so that the spindle could be approximated as a cylindrical surface enclosing the blades [26-28]; see dashed line in Figure 1(a). This meant that the material was sheared in the annular gap between the spindle and the outer wall. Assuming that the fluid is non-thixotropic, that the flow is quasi-steady and purely azimuthal, and that the azimuthal velocity only depends on the radius $r$, the shear stress and shear rate in the annular gap are given by [29]

$$
\begin{aligned}
& \tau(r)=\frac{M}{2 \pi r^{2} L_{\mathrm{tot}}}=\tau(R)\left(\frac{R}{r}\right)^{2}, \\
& \dot{\gamma}(r)=-r \frac{\mathrm{d} \omega}{\mathrm{d} r}
\end{aligned}
$$

respectively. We differentiate equation (2) with respect to $r$ and integrate equation (3) across the gap to obtain 


$$
\begin{gathered}
\frac{\mathrm{d} \tau(r)}{\mathrm{d} r}=-\frac{2}{r} \frac{M}{2 \pi r^{2} L_{\mathrm{tot}}}=-2 \frac{\tau(r)}{r} \\
\left.\omega\right|_{r=R_{\text {cup }}}-\left.\omega\right|_{r=R}=-\int_{R}^{R_{\text {cup }}} \frac{\dot{\gamma}(r)}{r} \mathrm{~d} r .
\end{gathered}
$$

We recall that the outer cup is stationary, $\left.\omega\right|_{r=R_{\text {cup }}}=0$, and interpret equation (4) as a change of variables between $\tau$ and $r$. This turns equation (5) into a Volterra integral equation of the first kind,

$$
\omega=-\frac{1}{2} \int_{\tau^{\text {in }}}^{\max \left(\tau^{\text {out }}, \tau_{\text {yield }}\right)} \frac{\dot{\gamma}(\tau)}{\tau} \mathrm{d} \tau,
$$

which relates the rotational speed of the spindle $\omega$ to the shear stress $\tau$ across the gap, where the unknown shear rate $\dot{\gamma}$ is a function of the shear stress. The upper integration limit is the larger of either the shear stress on the outer wall of the cup, calculated from equation (2) with $r=R_{\text {cup }}$, or the yield stress. The lower integration limit is the shear stress at the spindle, given by equation (1). Equation (6) can be solved numerically for the unknown shear rate function $\dot{\gamma}(\tau)$ for a given set of Couette viscometry data, without having to make any a priori assumption about the rheological model [23]. We note that the yield stress $\tau_{\text {yield }}$ is unknown and has to be determined as part of the solution. This requires another condition, which is that the shear rate vanishes at the yield stress, $\dot{\gamma}\left(\tau_{\text {yield }}\right)=0$.

\section{RESULTS}

We begin by showing in Figure 2 a typical example of rheological properties of tempered chocolate, measured for a cycle period of $60 \mathrm{~s}$. We present the first cycle of a single experiment, where the measured shear stress (angular velocity) is plotted as a function of angular velocity (shear stress) in Figures $2(a),(c)$, respectively. We use squares/triangles to differentiate the measurements during the upward/downward parts of the cycle. In both cases the shear stress (angular velocity) exhibits monotonic growth with angular velocity (shear stress). However, there is a small hysteresis between the upward and downward curves, which is illustrated by the orange shaded area in Figures 2(a) and $(c)$ and which persists in subsequent cycles. This is indicative of slight thixotropy although the difference between 


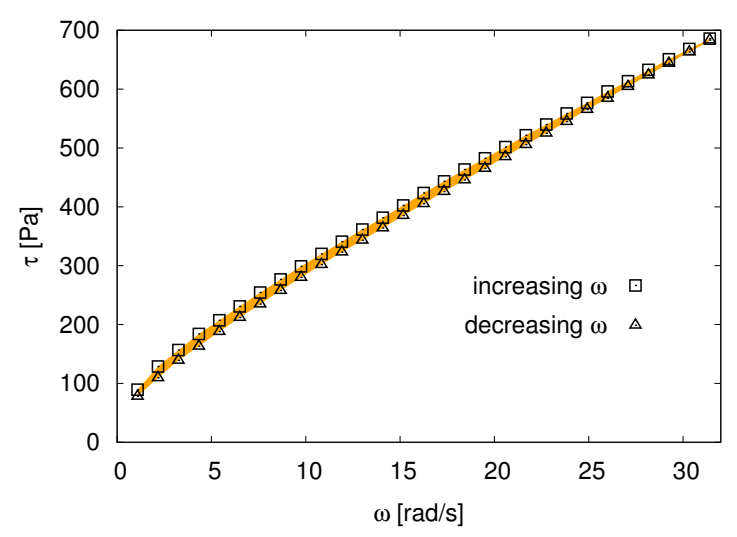

(a)

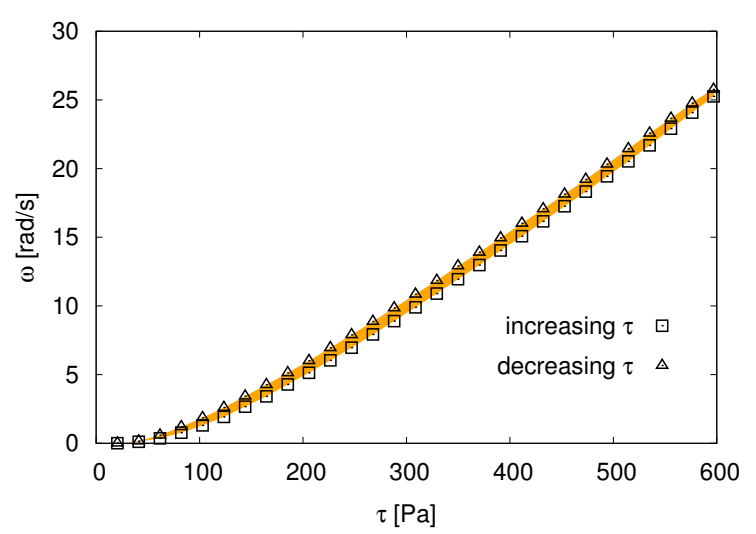

(c)

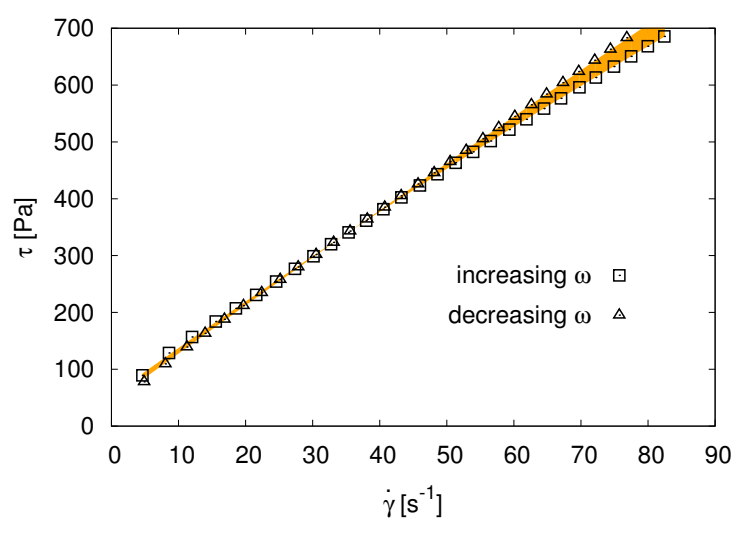

(b)

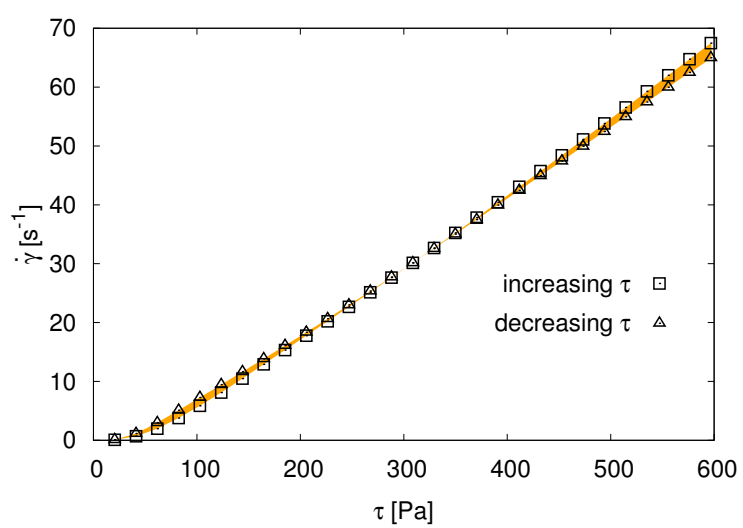

(d)

FIG. 2. Rheological measurements for a cycle period of $60 \mathrm{~s}$. Results shown for the first cycle with (a) shear stress as a function of the imposed angular velocity and (c) angular velocity as function of the imposed shear stress. The shaded area is the hysteresis area between the curves recorded during the upward/downward parts of the cycle. The processed data is shown in $(b)$ as shear stress as function of shear rate and in $(d)$ as shear rate as a function of shear stress.

the upward and the downward parts of the cycle is less than $6 \%$, which is comparable to that observed by Aeschlimann and Beckett [30] for untempered chocolate.

The corresponding processed data in Figures $2(b)$ and $(d)$ indicates that the shear stress (shear rate) also increases monotonically with shear rate (shear stress) during both the upward and downward parts of the cycle. However the shape of the processed curve $\tau(\dot{\gamma})$ differs from that of the unprocessed data $\tau(\omega)$ because of the integrated nonlinear relationship between $\omega$ and $\tau$ (Eq. 6). This has a clear impact on the width of the orange hysteresis region in Figures 2(b), (d), which increases for the larger shear rates (shear stresses), respectively, in contrast with the unprocessed data where the hysteresis region is almost uniform over the 
parameter range investigated. For small imposed shear stresses we measure zero shear rates, which implies the existence of a yield stress, while for larger shear stresses (shear rates) the relationship between $\dot{\gamma}$ and $\tau$ becomes approximately linear, suggesting that the chocolate reaches a constant viscosity. We now turn to the study of yielding at small values of the imposed stress in §III A, before discussing the constitutive behaviour of tempered chocolate in $\S I I I B$.

\section{A. Yielding}

\section{Experimental measurements}

To explore yielding, we cyclically imposed shear stress on the long spindle up to a maximum of $350 \mathrm{~Pa}$ with a period of $t_{\text {cycle }}=240 \mathrm{~s}$. As mentioned in $\S \mathrm{II} \mathrm{B}$, we subjected the sample to only three cycles of increasing and decreasing shear stress because at larger times the tempered state altered through further crystallisation. The measured shear rate is shown in the left column of Figure 3 on a log-log scale. The red arrows illustrate the direction of increasing and decreasing imposed stress. Here we focus on the experimental data points shown with symbols; a discussion of the computed lines follows in §III A 3.

When the shear stress is increased in the first cycle, non-zero shear rates indicative of deformation are measured for stresses as low as $12 \mathrm{~Pa}$. The monotonic growth of the shear rate with applied shear stress is weak for the lowest values of $\tau$, but increases rapidly before settling on a stress-strain rate curve with a slope of approximately one. This part of the curve is approximately retraced during the downward part of the cycle. In fact, in all three cycles, shear rates recorded during the upward and downward parts of the cycle overlap beyond a threshold value of the applied shear stress, which ranges from $40 \mathrm{~Pa}$ in the first cycle (consistent with the data shown in Figure 2(d)) to $110 \mathrm{~Pa}$ in the third cycle. In the first cycle, the downward curve separates from the upward curve when the shear stress is decreased to the lowest value of applied stress for which a non-zero shear rate could be resolved, which is approximately $40 \mathrm{~Pa}$. This is a good estimate of the dynamic yield stress, because it means that the chocolate comes to rest within a further decrement in stress of $3 \mathrm{~Pa}$, which also implies that the downward curve must steepen considerably around this value. 


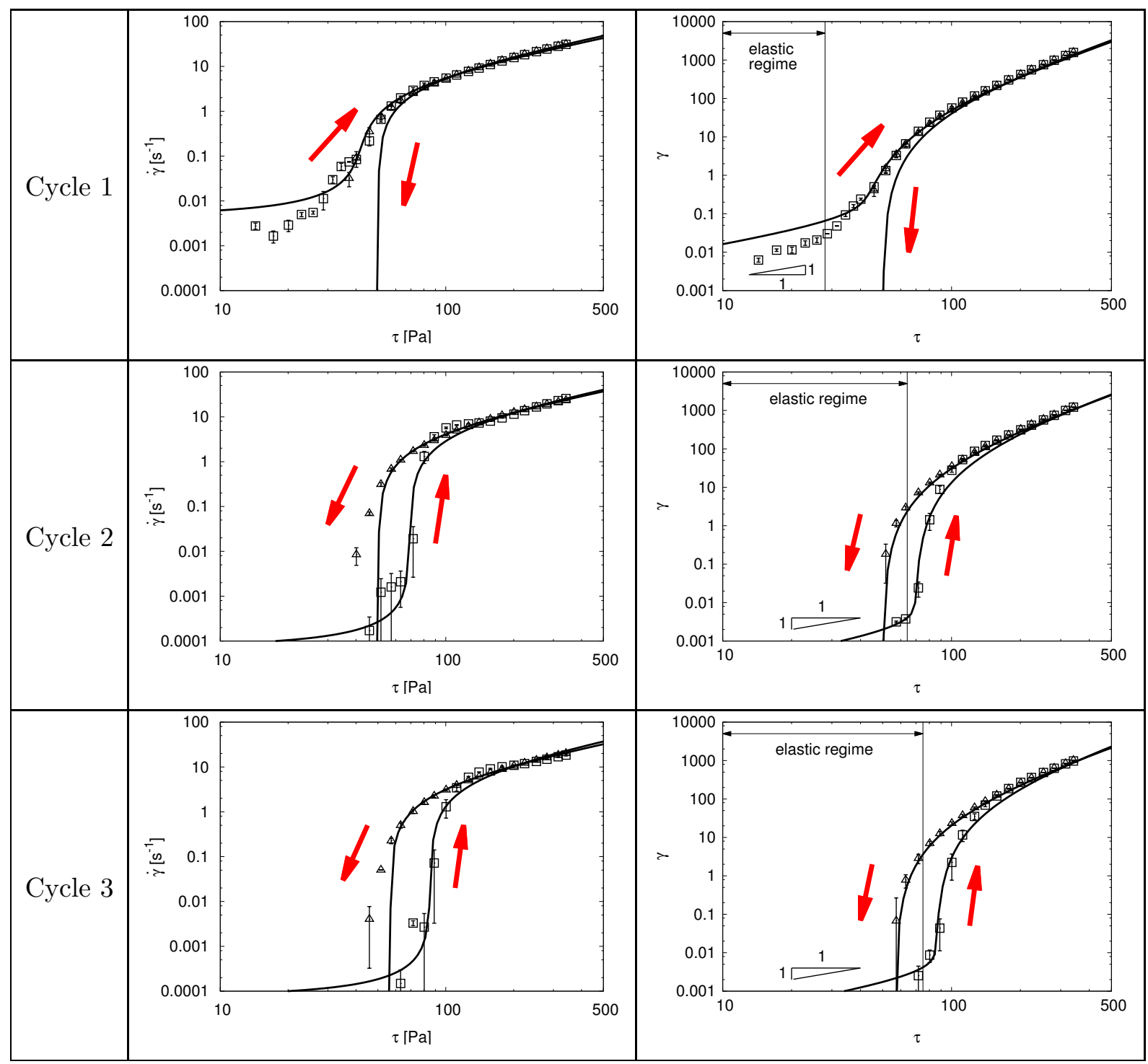

FIG. 3. Logarithmic plots of the shear rate (left column) and the shear strain (right column), $\gamma=\int \dot{\gamma} \mathrm{d} t$, as function of the shear stress, $\tau$, for successive cycles of increasing and decreasing $\tau$ with a cycle period of $t_{\text {cycle }}=240 \mathrm{~s}$. Points with errorbars represent experimentally measured values, while solid lines illustrate the results of matching computations with the White-Metzner model. Red arrows indicate the direction of increasing/decreasing shear stress.

The dynamic yield stress thus estimated varies weakly across the three cycles, from $38 \mathrm{~Pa}$ in the first cycle to $45 \mathrm{~Pa}$ in the third, as can be seen in Figure 3. In contrast, the shear rate curves obtained when increasing the shear stress evolve considerably across the three cycles in that the minimum value of $\tau$ for which deformation first occurs increases significantly, so that the upward curves shift to the right of the downward curves in both cycles 2 and 3 . 
This means that the value of apparent static yield stress is larger than the mininum value required to sustain flow, a phenomenon linked to the transient nature of flow for low shear stresses $[4,5,21]$.

In order to differentiate between elastic deformation and flow, we calculate the accumulated strain through time-integration of the shear rate, $\gamma=\int \dot{\gamma} \mathrm{d} t$, using the unsheared sample configuration, $\gamma=0$, as reference. The integrated shear strain is shown as a function of shear stress in the right column of Figure 3 on a log-log scale. Again, we initially focus on the experimental measurements shown with symbols. When incrementing $\tau$ from zero in cycle 1, the integrated shear strain exhibits an approximately linear variation with applied shear stress, which suggests a regime of elastic deformation so that $\gamma=1 / G \tau$, where $G$ is the shear modulus. The rapid steepening of the stress-strain curve for intermediate values of the applied shear stress signals the transition to a regime in which the material flows. We note that this transition from an elastic regime to a viscous regime resembles the rheological behaviour of wet soil, also a dense suspension, which was characterised by Ghezzehei and Or [31]. The largest integrated strain values in the elastic regimes highlighted in Figure 3 decrease by more than an order of magnitude from cycle 1 to cycle 2 , and then remain approximately constant between cycle 2 and cycle 3 . In fact, only two data points exceeding the resolution threshold of the rheometer could be captured in each of the latter two cycles, and these were for the largest values of stress within the elastic regime. Hence, the shear modulus of the material also increases by more than an order of magnitude from cycle 1 to cycle 2 . This suggests that the initial fluidisation of the material in cycle 1 is associated with significant reorganisation on the microscopic scale, an issue we will return to in §III A 3.

By contrast, the curves recorded during the decreasing part of the cycle remain very similar. In each cycle the material is brought to rest below $\tau_{\text {yield }} \simeq 40 \mathrm{~Pa}$, and there were no measurable values of elastic integrated strain values. The apparent absence of integrated strain values below $\tau_{\text {yield }} \simeq 40 \mathrm{~Pa}$ is attributed to the significant rise in shear modulus following the initial fluidisation of the material in the first cycle, so that elastic integrated strain could not be resolved by the rheometer. 


\section{Theoretical model}

The rheological behaviour of chocolate is commonly modelled using generalised Newtonian constitutive models where the viscosity $\mu(\dot{\gamma})$ depends on the shear rate, and for which the deviatoric fluid stresses are given by $\boldsymbol{\tau}=2 \mu(\dot{\gamma}) \boldsymbol{\epsilon}$, where $\boldsymbol{\epsilon}$ is the shear rate tensor and $\dot{\gamma}$ a scalar measure (typically an invariant) of its magnitude. However, these models do not take into account the elastic behaviour around the yield point, which we observed experimentally. In order to account for elasticity we consider the White-Metzner [19] visco-elastic model given by,

$$
\boldsymbol{\tau}+\frac{\mu(\dot{\gamma})}{G} \nabla \vec{\tau}=2 \mu(\dot{\gamma}) \boldsymbol{\epsilon}
$$

where $G$ is the shear modulus, and

$$
\stackrel{\nabla}{\boldsymbol{\tau}}=\frac{\mathrm{D}}{\mathrm{D} t} \boldsymbol{\tau}-(\nabla \boldsymbol{u})^{T} \cdot \boldsymbol{\tau}-\boldsymbol{\tau} \cdot(\nabla \boldsymbol{u})
$$

is the upper-convected stress tensor, containing the material derivative $\mathrm{D} / \mathrm{D} t$ and the gradient of the fluid velocity $\nabla \boldsymbol{u}$. This model recovers the generalised Newtonian constitutive behaviour, $\boldsymbol{\tau}=2 \mu(\dot{\gamma}) \boldsymbol{\epsilon}$ for purely viscous behaviour in the limit $G \rightarrow \infty$; finite values of $G$ incorporate elastic behaviour into the model. We will demonstrate in §III B that the approximately linear relationship between shear rates and stresses at large imposed shear stresses (shear rates) is well described by the Bingham model [32], therefore we choose the generalised Newtonian constitutive model in (7) as

$$
\mu(\dot{\gamma})=\mu\left(2\left|\mathcal{I}_{2}\right|^{1 / 2}\right)=\mu_{B}+\frac{\tau_{\text {yield }}}{2\left|\mathcal{I}_{2}\right|^{1 / 2}},
$$

where $\mathcal{I}_{2}$ is the second invariant of the shear rate tensor $\boldsymbol{\epsilon}=\frac{1}{2}\left(\nabla \boldsymbol{u}+(\nabla \boldsymbol{u})^{T}\right), \mu_{B}$ is the Newtonian plateau viscosity in the limit of large shear rates, and $\tau_{\text {yield }}$ is the yield stress.

With $\boldsymbol{u}=u_{\phi}(r ; t) \boldsymbol{e}_{\phi}$, the only nontrivial component of the White-Metzner constitutive equation is

$$
\tau_{r \phi}+\frac{1}{G} \frac{\partial \tau_{r \phi}}{\partial t}\left(\mu_{B}+\frac{\tau_{\text {yield }}}{2 \epsilon_{r \phi}}\right)=2 \epsilon_{r \phi}\left(\mu_{B}+\frac{\tau_{\text {yield }}}{2 \epsilon_{r \phi}}\right),
$$

where we have used that the second invariant of the symmetric shear rate tensor is $\mathcal{I}_{2}=-\epsilon_{r \phi}^{2}$, and 


$$
\epsilon_{r \phi}=\frac{1}{2}\left(\frac{\partial u_{\phi}}{\partial r}-\frac{u_{\phi}}{r}\right)=\frac{r}{2} \frac{\partial}{\partial r}\left(\frac{u_{\phi}}{r}\right) .
$$

Solving equation (10) for the shear rate $\epsilon_{r \phi}(r, t)$ yields two solutions, only one of which is physically meaningful and given by

$$
\begin{aligned}
\epsilon_{r \phi}(r ; t)=\frac{1}{4 \mu_{B}}\left(\tau_{r \phi}\right. & \left.-\tau_{\text {yield }}+\frac{\mu_{B}}{G} \frac{\partial \tau_{r \phi}}{\partial t}\right) \\
& +\frac{1}{4 \mu_{B}}\left(\left(\tau_{r \phi}-\tau_{\text {yield }}+\frac{\mu_{B}}{G} \frac{\partial \tau_{r \phi}}{\partial t}\right)^{2}+4 \tau_{\text {yield }} \frac{\mu_{B}}{G} \frac{\partial \tau_{r \phi}}{\partial t}\right)^{\frac{1}{2}}=\frac{1}{2} \dot{\gamma} .
\end{aligned}
$$

We evaluate the shear rate at the inner cylinder, $\dot{\gamma}(\tau)=2 \epsilon_{r \phi}(r=R ; t)$ as a function of the shear stress which is given by $\tau=\tau_{r \phi}(r=R ; t)=M(t) / 2 \pi L R^{2}$ as in Eqn 1 . Using the imposed torque profile

$$
M(t)=\frac{M_{\max }}{\frac{1}{2} t_{\text {cycle }}} t
$$

we can write $\tau=\tau_{r \phi}(r=R ; t)=\alpha t$, where $\alpha=M_{\max } /\left(t_{\text {cycle }} \pi L R^{2}\right)$, so that we have

$$
\dot{\gamma}(\tau)=\frac{1}{2 \mu_{B}}\left(\tau-\tau_{\text {yield }}+\frac{\mu_{B} \alpha}{G}\right)+\frac{1}{2 \mu_{B}}\left(\left(\tau-\tau_{\text {yield }}+\frac{\mu_{B} \alpha}{G}\right)^{2}+\frac{4 \tau_{\text {yield }} \mu_{B} \alpha}{G}\right)^{\frac{1}{2}} .
$$

\section{Comparison between model and experiments}

The analytical solution of the White-Metzner model given by equation (14) was fitted to the experimental data from the left column of Figure 3, in order to determine the constitutive parameters $\tau_{\text {yield }}, \mu_{B}$ and $G$ for the up and down parts of the three cycles for which data was obtained. We used orthogonal distance regression (ODR) in order to account for

experimental errors on both stress and shear rate. This method minimises the distance between the model curve and each data point along the direction normal to the model curve, and we weighted each data point by the standard error in both stress and strain rate.

The values of $\tau_{\text {yield }}, \mu_{B}$ and $G$ obtained for each half cycle are presented in Table I. These fit parameters were then used to represent shear rate and integrated shear strain as a function of shear stress, with solid lines, in the left and right columns of Figure 3, respectively. The comparison between experiment and theory shows that the theoretical curves capture the main features of the experimental variations in shear rate and integrated 
shear strain as a function of applied stress. This indicates that the White-Metzner model provides an appropriate theoretical framework to interpret the experimental observations discussed in §III A 1. However, we note that in contrast with the fits of the Bingham model which we present in §III B, the White-Metzner curves shown in Figure 3 do not provide a close fit in terms of the goodness of fit parameter $\chi_{\nu}^{2}=\chi^{2} / \nu$, where $\chi^{2}$ is the weighted sum of squared deviations and $\nu$ the number of degrees of freedom, i.e. the number of data points with non-zero weights minus the number of model parameters.

\begin{tabular}{|c|c|c|}
\hline Cycle number & increasing $\tau$ & decreasing $\tau$ \\
\hline 1 & $\begin{array}{c}\tau_{\text {yield }}=41.7 \pm 0.3 \mathrm{~Pa} \\
\mu_{B}=10.66 \pm 0.07 \mathrm{~Pa} \mathrm{~s} \\
G=706 \pm 84 \mathrm{~Pa}\end{array}$ & $\begin{array}{c}\tau_{\text {yield }}=50.1 \pm 0.6 \mathrm{~Pa} \\
\mu_{B}=9.36 \pm 0.05 \mathrm{~Pa} \mathrm{~s} \\
G=O\left(10^{9}\right)\end{array}$ \\
\hline 2 & $\begin{array}{c}\tau_{\text {yield }}=69.3 \pm 0.8 \mathrm{~Pa} \\
\mu_{B}=10.71 \pm 0.08 \mathrm{~Pa} \mathrm{~s} \\
G=44,425 \pm 4,609 \mathrm{~Pa}\end{array}$ & $\begin{array}{c}\tau_{\text {yield }}=50.2 \pm 0.4 \mathrm{~Pa} \\
\mu_{B}=12.18 \pm 0.06 \mathrm{~Pa} \mathrm{~s} \\
G=O\left(10^{9}\right)\end{array}$ \\
\hline 3 & $\begin{array}{c}\tau_{\text {yield }}=85.3 \pm 1.6 \mathrm{~Pa} \\
\mu_{B}=11.21 \pm 0.31 \mathrm{~Pa} \mathrm{~s} \\
G=43,261 \pm 6,319 \mathrm{~Pa}\end{array}$ & $\begin{array}{c}\tau_{\text {yield }}=56.8 \pm 1.9 \mathrm{~Pa} \\
\mu_{B}=13.75 \pm 0.15 \mathrm{~Pa} \mathrm{~s} \\
G=O\left(10^{9}\right)\end{array}$ \\
\hline
\end{tabular}

TABLE I. Fitted parameters of the White Metzner model.

The model indicates that the considerable differences between the shear rate curves for the "up" parts of cycles 1 and 2 are associated with an increase in yield stress of more than $60 \%$, from approximately $\tau_{\text {yield }}=42 \mathrm{~Pa}$ in the first half cycle to $69 \mathrm{~Pa}$ in the second. More significantly, however, the shear modulus increases by a factor of more than 60, from $G=706 \mathrm{~Pa}$ to $G=44,425 \mathrm{~Pa}$. In the third half cycle, the shear modulus retains a value similar to that of the second one, while the yield stress rises further to $\tau_{\text {yield }}=85 \mathrm{~Pa}$. We note that the values for $G$ in the second and third cycles are larger by a factor of approximately 2.5 compared with those of Taylor et al. [12] for untempered chocolate. Hence, elastic strains are much larger in the first cycle than in subsequent ones. This explains why elastic strains are resolved experimentally for much lower values of the applied stress in the first half cycle 
compared with subsequent cycles where only two experimental data points could be captured within the elastic regime.

The cycle-to-cycle increase of both yield stress and shear modulus may be explained by considering the microscopic structure of tempered chocolate, which is dominated by the interaction between suspended cocoa particles and the distribution of crystals in the material. The increase of applied shear stress during the first half cycle follows a pre-shearing procedure (§II B) aimed at preserving the microscopic structure of the chocolate developed during the tempering process. Hence, at this stage the cocoa particles in the chocolate are randomly distributed and particle networks are weak. This results in a moderate value of the yield stress, when measured for increasing applied shear stresses. Moreover, the weakness of the particle networks is associated with a small resistance to elastic deformation, which concurs with the small value of the shear modulus measured in the first cycle. The networks start to break at the yield point, increasing the propensity of individual particles to move. Cocoa particles and cocoa butter crystals align under shear to form structures [33]. Upon reduction of the shear stress in the downward half of the cycle, these ordered flow structures lead to the formation of particle networks with significantly increased strength, resulting in increasing values of both the yield stress and the shear modulus. In contrast, the Newtonian plateau viscosity obtained from the fits of the upward half cycles 2 and 3 remains largely unchanged with $10.7 \mathrm{~Pa} \mathrm{~s} \lesssim \mu_{B} \lesssim 11.2 \mathrm{~Pa}$ s. This is because the particle and crystal networks are essentially broken down in flow and thus they do not impact the viscosity of the yielded chocolate for large values of the shear rate. The continued increase of the static yield stress from cycle 2 to 3 may also be influenced by unavoidable crystallisation over time that raises the temper index (see $\S I I)$.

Based on these microscopic considerations, we expect the value of the shear modulus in the downward half of the cycle to be similar to that obtained in the upward cycles 2 and 3 . The very large values of $G \sim 10^{9} \mathrm{~Pa}$ shown in Table I are somewhat questionable because of the absence of measurements in the elastic regime in the downward halves of the cycles. This is because the yield stress in the downward half of the cycles $\tau_{\text {yield }} \simeq 50 \mathrm{~Pa}$ s is smaller than in the upward halves of cycles 2 and 3, so that the values of the elastic strain when $G \simeq 4.4 \times 10^{4} \mathrm{~Pa}$ falls below the resolution threshold of the rheometer for $\tau \lesssim 50 \mathrm{~Pa}$. We attribute the larger variation in the downward parts of the cycles of the viscosity, 9.36 $\mathrm{Pa} \mathrm{s}$ $\leq \mu_{B} \leq 13.75 \mathrm{~Pa} \mathrm{~s}$, to the absence of data points in the elastic regime which renders the fit 
(a)

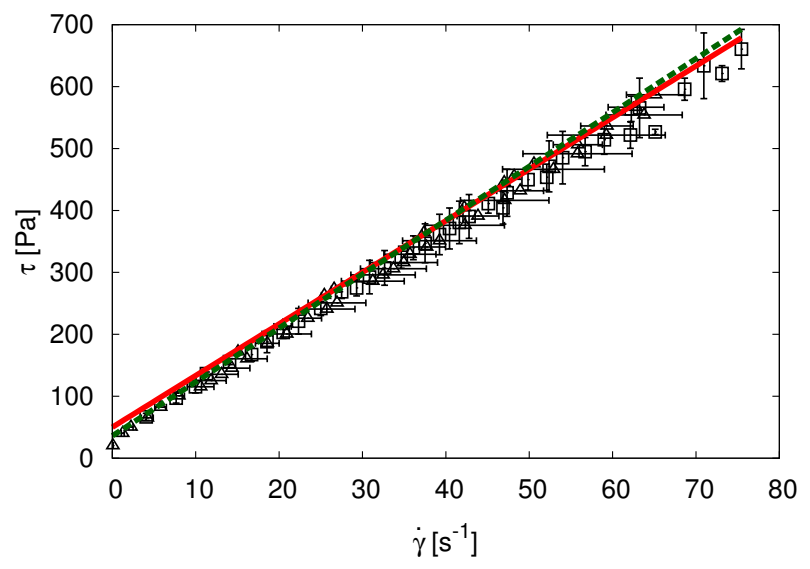

(b)

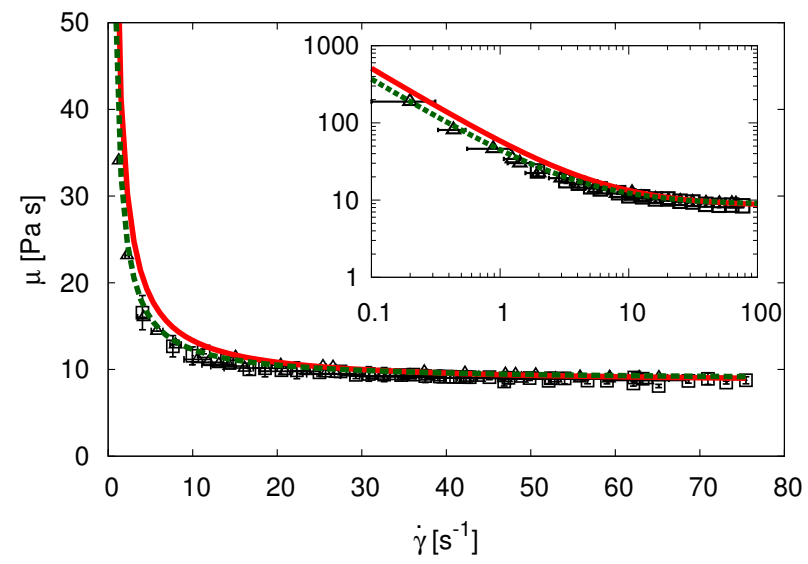

FIG. 4. Constitutive behaviour of tempered chocolate as determined in the first cycle for decreasing shear rates (squares) and shear stress (triangles). Part (a) shows the shear stress, $\tau$, as a function of shear rate, $\dot{\gamma}$, while $(b)$ shows the corresponding viscosity, $\mu$, as a function of $\dot{\gamma}$. Inset: $\log -\log$ plot of the same graph. In the interest of readability we only plot approximately one quarter of the actual data points. The dashed green lines correspond to a two-parameter fit of the Bingham model, while the solid red lines indicate a fit of the Bingham model where the value of the yield-stress is set to $\tau_{\text {yield }}=50.1 \mathrm{~Pa}$, the value we determined in $\S I I I \mathrm{~A}$.

of the model solution to the experimental measurements less accurate.

The dynamic yield stress obtained from the downward parts of the cycles remains approximately constant across the three cycles, but it is slightly larger than the value of $40 \mathrm{~Pa}$ estimated directly from the experimental measurements in §IIIA1. This is because the theoretical fit does not accurately capture the two data points with the smallest applied shear stress in cycles 2 and 3. This discrepancy between model and experiment suggests that the particle and crystal networks may form progressively as the applied shear stress is decreased resulting in less rapid increases in viscosity towards the yield point than implied by the Bingham model.

\section{B. Flow behaviour}

We showed in §III A 3 that elastic deformation contributes to the rheology of tempered chocolate near the yield point, and found that the constitutive behaviour of yielded tempered chocolate is well approximated by a Bingham model. We now extend the range of shear stresses by a factor of two in order to identify the generalised Newtonian model that 
most accurately describes the experimental flow behaviour, and determine the associated constitutive parameter values. Figure $4(\mathrm{a}, \mathrm{b})$ shows shear stress and viscosity plotted as a function of shear rate. The data corresponds to an extensive set of experiments, performed by imposing either the shear rate or the shear stress, for cycle periods of $t_{\text {cycle }}=30 \mathrm{~s}$, $60 \mathrm{~s}, 120 \mathrm{~s}$ and $240 \mathrm{~s}$ (as described in $\S I I \mathrm{~B}$ ). We only consider data recorded during the downward part of the first cycle in each experiment, in order to circumvent the influence of both elasticity and thixotropy near the yield point. All the rheological data fall onto a single flow curve, and thus we calculate the mean data across all cycle periods. In order to avoid overlapping data obscuring the plot, we only show approximately one quarter of the actual data points; the error bars correspond to the standard error across all the data sets. The consistent overlap between the constitutive curves obtained by imposing shear rate (squares) and shear stress (triangles), respectively, suggests that our measurements are robust and reproducible. Figure 4(b) shows that the viscosity of the tempered chocolate decreases smoothly towards a constant value, which is reached for $\dot{\gamma} \gtrsim 40 \mathrm{~s}^{-1}$. This decrease of viscosity is indicative of shear-thinning rheology, with a constant viscosity limit of a Newtonian plateau, previously observed by Briggs and Wang [15] for $\dot{\gamma}>70 \mathrm{~s}^{-1}$.

We note that we also attempted to fit the Casson model to our data but were unable to capture the experimental data near the yield point. Whereas the experimental data exhibits a sharp solid-liquid transition close to the yield point, the Casson model predicts a gradual transition. This significant limitation of the Casson model has been previously highlighted for untempered chocolate by Prentice et al. [18]. Finally we considered a fit of the Herschel-Bulkley model, a generalisation of the Bingham model to arbitrary values of the power index $n$. The best fit of this three-parameter model to the experimental data gave $n=1.02 \pm 0.006$, which indicates that the Bingham model, which has $n=1$, is most appropriate to represent our data.

A two-parameter fit of the Bingham model to the experimental data shown in Figure 4 is shown with a dashed green line. The solid red line corresponds to a one-parameter fit $\left(\chi_{\nu}^{2}=5.6\right)$, where we prescribed the yield stress value as $\tau_{\text {yield }}=50.1 \mathrm{~Pa}$, previously obtained in §III A 2. The two-parameter fit provided the closest match to the data $\left(\chi_{\nu}^{2}=1.7\right)$ and gave $\tau_{y}=36.1 \pm 0.6 \mathrm{~Pa} ; \mu_{B}=8.7 \pm 0.02 \mathrm{~Pa}$ s, while the one-parameter fit gave a similar value of $\mu_{B}=8.33 \pm 0.02 \mathrm{~Pa}$. The wider range of shear stress (shear rate) evaluated here is likely to yield a better estimate of the Newtonian plateau viscosity than in §III A 3. 


\section{SUMMARY}

We have characterised the flow curve and yield behaviour (on moderate time scales) of molten tempered milk chocolate using rheological measurements performed with a rotational rheometer fitted with a four-bladed vane spindle in a large-gap setup. We have found that the Bingham model provides the best fit to our extensive flow curve data. We have also shown that the yield behaviour can be captured by the viscoelastic White-Metzner model, which has an analytical solution when the Bingham model provides the relationship between stress and strain rate. The solution indicates that a noticeable elastic deformation precedes flow as the shear stress is increased. Following an initial fluidisation cycle, the shear modulus of the tempered chocolate increases by a factor sixty which is consistent with changes to the microscopic structure of the material due to shear-induced alignment of particles in the fluidised phase. The constitutive parameters determined experimentally may in turn be incorporated in a viscoelastic or generalised Newtonian model to explore the deformation and flow of molten chocolate. A paper is currently in preparation that utilises these parameters to characterise the fluidisation of molten chocolate due to vertical mechanical vibrations - a process commonly employed on industrial production lines - by direct comparison between experiments and numerical simulations.

\section{ACKNOWLEDGMENTS}

NB is grateful to Mondelez International and the School of Mathematics (The University of Manchester) for funding this work.

[1] P. Coussot, Rheometry of pastes, suspensions, and granular materials: applications in industry and environment (John Wiley \& Sons, 2005).

[2] N. J. Balmforth, I. A. Frigaard, and G. Ovarlez, Annu. Rev. Fluid Mech. 46, 121 (2014).

[3] R. G. Larson, The structure and rheology of complex fluids (Oxford University Press, New York, 1999).

[4] G. Ovarlez, S. Cohen-Addad, K. Krishan, J. Goyon, and P. Coussot, J. Non-Newton. Fluid Mech. 193, 68 (2013). 
[5] T. Divoux, , C. Barentin, and S. Manneville, Soft Matter 7, 8409-8418 (2011).

[6] S. T. Beckett, The science of chocolate (Royal Society of Chemistry, 2000).

[7] E. Afoakwa, A. Paterson, M. Fowler, and J. Vieira, Int. J. Food Sci. Technol. 44, 162 (2009).

[8] P. Coussot, Q. Nguyen, H. Huynh, and D. Bonn, J. Rheol. 46, 573 (2002).

[9] J. Chevalley, J. Texture Stud. 6, 177 (1975).

[10] E. Gonçalves and S. Lannes, Ciência e Tecnologia de Alimentos 30, 845 (2010).

[11] C. Servais, H. Ranc, and I. Roberts, J. Texture Stud. 34, 467 (2004).

[12] J. Taylor, I. Van Damme, M. Johns, A. Routh, and D. Wilson, J. Food Sci. 74, E55 (2009).

[13] L. Wilson, R. Speers, and M. Tung, J. Texture Stud. 24, 269 (1993).

[14] K. Smith, "Chocolate tempering," (2006), Confectionery Manufacturing Expo, Brussels (https://courses.ecolechocolat.com/lobjects/pdf/belgium_tempering.pdf).

[15] J. Briggs and T. Wang, J. Am. Oil Chem. Soc. 81, 117 (2004).

[16] R. B. Beckett, Industrial chocolate manufacture and use (Chapman \& Hall, New York, fourth edition, 2009).

[17] B. Minifie, Chocolate, cocoa and confectionery: science and technology (Springer Science \& Business Media, 2012).

[18] J. Prentice et al., Measurements in the rheology of foodstuffs (Elsevier Applied Science Publishers Ltd., 1984).

[19] J. White and A. Metzner, J. Appl. Polym. Sci. 7, 1867 (1963).

[20] M. Dinkgreve, M. Denn, and D. Bonn, Rheol. Acta 56, 189 (2017).

[21] G. Ovarlez and X. Chateau, Phys. Rev. E 77, 061403 (2008).

[22] Q. Nguyen and D. Boger, Annu. Rev. Fluid Mech. 24, 47 (1992).

[23] Y. Yeow, W. Ko, and P. Tang, J. Rheol. 44, 1335 (2000).

[24] A. G. F. Stapley, H. Tewkesbury, and P. J. Fryer, JAOCS 76, 677 (1999).

[25] J. van Wazer, J. Lyons, K. Kim, and R. Colwell, Viscosity and flow measurement: a laboratory handbook of rheology (Interscience Publishers, 1963).

[26] N. Alderman, G. Meeten, and J. Sherwood, J. Non-Newton. Fluid Mech. 39, 291 (1991).

[27] P. Liddel and D. Boger, J. Non-Newton. Fluid Mech. 63, 235 (1996).

[28] Q. Nguyen and D. Boger, J. Rheol. 27, 321 (1983).

[29] Q. Nguyen and D. Boger, Rheol. Acta 26, 508 (1987).

[30] J. Aeschlimann and S. Beckett, J. Texture Stud. 31, 541 (2000). 
[31] T. A. Ghezzehei and D. Or, Soil Sci. Soc. Am. J. 65, 624 (2001).

[32] E. C. Bingham, Fluidity and plasticity, Vol. 2 (McGraw-Hill New York, 1922).

[33] E. Windhab, in Physico-chemical aspects of food processing (Springer, 1995) pp. 80-116. 